\title{
Comparison of the prevalence of incidental and non-incidental papillary thyroid microcarcinoma during 2008-2016: a single-center experience
}

\author{
Krzysztof Kaliszewski ${ }^{*}$ (D), Agnieszka Zubkiewicz-Kucharska², Paweł Kiełb', Jerzy Maksymowicz¹,
}

Aleksander Krawczyk ${ }^{1}$ and Otto Krawiec ${ }^{1}$

\begin{abstract}
Background: The incidence of papillary thyroid microcarcinoma (PTMC) is increasing; however, it is not clear whether this reflects an increase in the incidence of incidental or in that of non-incidentally (presurgically) discovered PTMC (IPTMC vs. NIPTMC). We assessed the incidence of IPTMC and NIPTMC over the past 9 years, to discern whether the increase in PTMC incidence is due to improved diagnostics or to a real increase in the incidence.

Methods: We performed a retrospective chart review of 4327 patients who were consecutively admitted to and surgically treated for thyroid pathology at a single institution. As a main presurgical diagnostic test, all patients underwent ultrasound-guided fine-needle aspiration biopsy (UG-FNAB). The analyzed time frame was divided into three equal periods (I: 2008-2010, II: 2011-2013, III: 2014-2016), and IPTMCs and NIPTMCs were assessed and compared in each period.

Results: We evaluated 393 (9.08\%) patients with thyroid malignancy, of which 156 (3.60\% of all thyroid tumors [Ts]; $39.69 \%$ of all thyroid cancers [TCs]) were diagnosed as PTMC. The prevalence of NIPTMC among all TCs increased from $16.66 \%$ in 2008 to $33.75 \%$ in 2016, while that of IPTMC decreased from 20.83\% in 2008 to $13.75 \%$ in 2016. The incidence rates of NIPTMC and IPTMC in period III differed statistically significantly $(p<0.0001)$. The prevalence rate of NIPTMC in period III was higher than that in period II, yet comparable to that in period I ( $p=0.0014 ; p=0.2804$, respectively).
\end{abstract}

Conclusions: The prevalence of NIPTMC, rather than that of IPTMC, is escalating; this may be due to better presurgical diagnosis.

Keywords: Incidental, Non-incidental, Papillary thyroid microcarcinoma

\section{Background}

Thyroid carcinoma (TC) is the most common malignant tumor of the endocrine system [1]. It constitutes approximately $1 \%$ of all human malignancies and is the main cause of death among endocrine tumor-related deaths [2]. In 2010, Jemal et al. reported 44,700 new cases of thyroid cancers per year, worldwide, and 1700

\footnotetext{
* Correspondence: krzysztofkali@wp.pl

${ }^{1}$ First Department and Clinic of General, Gastroenterological, and Endocrine Surgery, Wroclaw Medical University, 66 Maria Skłodowska-Curie Street, 50-369 Wrocaw, Poland

Full list of author information is available at the end of the article
}

deaths due to this condition occurred annually [3]. An annual increase of $5.3 \%$ in TC incidence was reported by Magreni et al. in 2015 [4].

Papillary thyroid cancer (PTC), which is the main type of TC, accounts for $80 \%$ of all thyroid malignancies [5]. The prognosis of PTC is generally favorable; however, for some subtypes, prognosis may depend on the cancer stage at the time of diagnosis. For early stages, the 10-year survival rate reaches $90 \%$, whereas for later stages, the 10-year survival rate is not as high [5].

A clinically important type of PTC is a tumor with a small size (diameter $\leq 1.0 \mathrm{~cm}$ ), regardless of whether 
lymph node and local invasion, as well as distant metastases, has occurred. The World Health Organization (WHO) defines these tumors as a papillary thyroid microcarcinoma (PTMC) [6]. PTMCs account for approximately $30 \%$ of all PTCs [7]. Some authors have described PTMCs as tumors with low malignancy, which are slow-growing, minimally invasive, and associated with low mortality [8]. Based on autopsy studies, Solares et al. have confirmed that up to $36 \%$ of PTMCs had low aggressiveness [9]. Other reports have also described these tumors as common and typical findings, with a favorable prognosis [10]. However, very aggressive forms of PTMC have also been described [11]. Therefore, Gao et al. stated that PTCs with a small size $(\leq 1.0 \mathrm{~cm}$ in diameters) do not always behave as indolent tumors [12].

To date, the diagnosis of PTMC has been highly reliant on a high-frequency ultrasonography examination and ultrasound-guided fine-needle aspiration biopsy (UG-F$\mathrm{NAB}$ ), which remains the standard diagnostic procedure for the evaluation of thyroid nodules. The main purpose of UG-FNAB is to distinguish between malignant and benign tumors and to identify patients requiring surgical treatment [13].

The accuracy of diagnostic procedures for thyroid nodules has improved over the last few years, and the ease of access to such diagnostic modalities may be the reason for the higher prevalence of thyroid tumors. However, the exact cause of this increase is still debated. As we have observed a continuous increase in the PTMC incidence over a number of years, we have contemplated various reasons for this phenomenon. Although improvements in the imaging tools, availability of UG-FNAB, and easier access to diagnostic thyroid pathology may play a role, some changes in the environment may also have caused a real increase in the morbidity rate.

The purpose of this study was to assess and compare the incidence of PTMC over the last 9 years and to identify whether there is a difference in the number of presurgically discovered (non-incidental) and non-discovered (incidental) PTMCs, to determine whether the increased incidence in PTMC is due to improved diagnostics (mainly UG-FNAB).

\section{Methods}

We performed a retrospective chart review of 4327 patients who were consecutively admitted to and surgically treated for thyroid pathology at a single institution, from January 1, 2008, to December 31, 2016. The analyzed time frame was divided into three equal periods (period I: 2008-2010, period II: 2011-2013, period III: 20142016). Next, we assessed and compared the incidence rates of incidental PTMCs (IPTMCs) and non-incidental PTMCs (NIPTMCs) in these three periods. All of the ultrasound examinations were performed by the same team of radiologists experienced in thyroid sonography.
All patients underwent UG-FNAB as the main presurgical diagnostic test. The same equipment and ultrasonography set in all biopsies were used. A $10-\mathrm{MHz}$ linear probe of ultrasonography set has been applied. The UG-FNAB was performed using $0.5-\mathrm{mm}$ gauge needles and 10-cc syringes for each procedure. Clinical and pathological classification was performed according to the TNM classification criteria (7th Edition, 2015) by the American Joint Committee on Cancer (AJCC) [14]. All of the patients underwent total thyroidectomy by the same team of surgeons experienced in thyroid surgery, and all histopathological specimens were examined by the same two pathologists, who were both experienced in diagnosing thyroid malignancy.

\section{Statistical analysis}

Statistical analysis was conducted with the use of Statistica vs. 12 (StatSoft, Inc., Tulsa, OK, USA; 2014). The following statistical measures were used: arithmetical mean (x), median and standard deviation (SD), and ranges of determined parameters in study groups.

The Shapiro-Wilk test was used to confirm the normality of data distribution. As data demonstrated a normal distribution, $t$ tests were used to assess the significance of differences. Intergroup frequency assessment was performed using a chi-squared test. Yate's correction was applied when the expected frequency was less than 5 or the total count was less than 50 .

$P$ values $<0.05$ were taken as indicating statistically significant differences, while $p$ values from 0.05 to $<$ 0.10 were considered as indicating borderline statistical significance.

\section{Results}

From 4327 patients diagnosed for thyroid pathology at our institute during the study period, we evaluated 393 (9.08\%) patients with thyroid malignancy, of whom 156 (3.60\% of total thyroid pathology; $39.69 \%$ of TC patients) were diagnosed with PTMC. In this homogenous group, there were 52 (33.33\%) patients with IPTMC and 104 (66.67\%) with NIPTMC. There were 45 (89.18\%) females and 7 (10.82\%) males in the IPTMC group and 98 (95\%) females and 6 (5\%) males in the NIPTMC group $(p=0.1013)$. The mean age of all patients with PTMC was $48.6( \pm 14.6)$, and 44.6 $( \pm 15.9)$ and $49.0( \pm 14.5)$ for males and females, respectively $(p=0.1390$; Table 1$)$.

The prevalence of PTMC increased over the 9-year period, from $37.5 \%$ in 2008 to $47.5 \%$ in 2016 ( $p=$ 0.0414). The prevalence of NIPTMC increased from $16.66 \%$ of all TCs in 2008 to $33.75 \%$ in 2016, but IPTMC decreased from $20.83 \%$ in 2008 to $13.75 \%$ in 2016 (Table 2). In the years 2009, 2015, and 2016, we observed more patients with NIPTM than with IPTMC (Table 3; Fig. 1a, b and c). Moreover, we noticed a 
Table 1 Demographic characteristics of patients with a diagnosis of papillary thyroid microcarcinoma (PTMC)

\begin{tabular}{ll}
\hline Parameter & PTMC $(n=156)$ \\
\hline Gender & $13(8.3 \%)$ \\
Male & $143(91.7 \%)$ \\
Female & \\
Age (years) & $48.6 \pm 14.6$ \\
All patients & $44.6 \pm 15.9$ \\
Male & $49.0 \pm 14.5$ \\
Female &
\end{tabular}

Descriptive data are presented as numbers ( $n)$, percentage (\%) and mean \pm standard deviation $( \pm \mathrm{SD})$

statistically significant predominance of NIPTMC over IPTMC in periods I (2008-2010) and III (2014-2016), but not in period II (2011-2013), ( $p=0.0216 ; p<0.0001$; $p=0.6176$. respectively; Table 4 , Fig. $1 \mathrm{~d}$, e, and f). Furthermore, there was an increase in the prevalence of NIPTMC in period III as compared to period II ( $p=$ $0.0014)$, but not as compared to period I $(p=0.2804)$ (Table 5).

\section{Discussion}

The incidence of PTMC has been increasing worldwide. In our study, we observed that the prevalence of this tumor has increased more than four times, from 1.86\% of all thyroid tumors in 2008 to $7.97 \%$ in 2016. Although it is typically minimally invasive, Gao et al. described PTMC as a public health concern because of its tremendous increase in the past few decades [12]. Additionally, it has been suggested that if any additional factors, such as extrathyroidal invasion, lymph node metastases, or the BRAF V600E mutation, are found, PTMC should be treated as a "larger" papillary thyroid cancer [15].
Table 3 The prevalence of incidental and non-incidental papillary thyroid microcarcinoma in each year

\begin{tabular}{lllll}
\hline & Incidental & Non-incidental & Total & $p$ \\
\hline 2008 & $5(1.03 \%)$ & $4(0.83 \%)$ & $9(1.86 \%)$ & 0.7115 \\
2009 & $3(0.79 \%)$ & $10(2.62 \%)$ & $13(3.40 \%)$ & 0.0261 \\
2010 & $3(0.71 \%)$ & $9(2.13 \%)$ & $12(2.84 \%)$ & 0.0578 \\
2011 & $4(0.83 \%)$ & $5(1.04 \%)$ & $9(1.86 \%)$ & 0.7169 \\
2012 & $9(1.66 \%)$ & $9(1.66 \%)$ & $18(3.31 \%)$ & 1 \\
2013 & $7(1.24 \%)$ & $9(1.59 \%)$ & $16(2.83 \%)$ & 0.5874 \\
2014 & $4(1.06 \%)$ & $6(1.60 \%)$ & $10(2.66 \%)$ & 0.4911 \\
2015 & $6(1.01 \%)$ & $25(4.21 \%)$ & $31(5.22 \%)$ & 0.0001 \\
2016 & $11(2.31 \%)$ & $27(5.66 \%)$ & $38(7.97 \%)$ & 0.003 \\
\hline
\end{tabular}

Statistically significant differences are shown in italics

Postoperatively, PTMC is often found in multinodular goiter (MNG) and it is then diagnosed as IPTMC [16]. In our study, the prevalence of IPTMC increased from $1.03 \%$ of all thyroid tumors in 2008 to $2.31 \%$ in 2016 , together with the increase of all PTMCs (from $1.86 \%$ in 2008 to $7.97 \%$ in 2016). In terms of total PTMC, the prevalence of IPTMC has decreased approximately by half: from $20.83 \%$ in 2008 to $13.75 \%$ in 2016 . Li et al. reported that IPTMC is undetectable before surgery, due to its coexistence with MNG, its small size, and its deep localization within thyroid gland [17]. For that reason, in MNG, every nodule should be assessed by ultrasound examination to determine the risk of cancer. In our previous study, we revealed that the assistance of a radiologist in UG-FNAB procedures increases the value of the procedure [18].

It was suggested that pre-operative diagnosis of PTMC is difficult and therefore rare, because of its slow growth rate, absence of specific symptoms, clinical characteristics, and potential co-occurrence with benign thyroid

Table 2 The prevalence of incidental and non-incidental papillary thyroid microcarcinoma (IPTMC and NIPTMC) according to all thyroid tumors and all thyroid cancers in years 2008-2016

\begin{tabular}{|c|c|c|c|c|c|c|c|c|c|}
\hline & 2008 & 2009 & 2010 & 2011 & 2012 & 2013 & 2014 & 2015 & 2016 \\
\hline \multicolumn{10}{|l|}{ For all thyroid tumors } \\
\hline IPTMC & $5(1.03 \%)$ & $3(0.79 \%)$ & $3(0.71 \%)$ & $4(0.83 \%)$ & $9(1.66 \%)$ & $7(1.24 \%)$ & $4(1.06 \%)$ & $6(1.01 \%)$ & $11(2.31 \%)$ \\
\hline NIPTMC & $4(0.83 \%)$ & $10(2.62 \%)$ & $9(2.13 \%)$ & $5(1.04 \%)$ & $9(1.66 \%)$ & $9(1.59 \%)$ & $6(1.60 \%)$ & 25 (4.21\%) & 27 (5.66\%) \\
\hline PTMC & $9(1.86 \%)$ & $13(3.40 \%)$ & $12(2.84 \%)$ & $9(1.86 \%)$ & 18 (3.31\%) & $16(2.83 \%)$ & $10(2.66 \%)$ & 31 (5.22\%) & 38 (7.97\%) \\
\hline All thyroid cancers & $24(4.96 \%)$ & $26(6.81 \%)$ & $36(8.51 \%)$ & 29 (6.00\%) & 51 (9.39\%) & $53(9.38 \%)$ & $32(8.51 \%)$ & $62(10.44 \%)$ & $80(16.77 \%)$ \\
\hline All thyroid tumors & $484(100 \%)$ & $382(100 \%)$ & $423(100 \%)$ & $483(100 \%)$ & $543(100 \%)$ & $565(100 \%)$ & $376(100 \%)$ & $594(100 \%)$ & 477 (100\%) \\
\hline \multicolumn{10}{|l|}{ For all thyroid cancers } \\
\hline IPTMC & $5(20.83 \%)$ & $3(11.53 \%)$ & $3(8.33 \%)$ & $4(13.79 \%)$ & $9(17.64 \%)$ & $7(13.20 \%)$ & $4(12.5 \%)$ & $6(9.67 \%)$ & $11(13.75 \%)$ \\
\hline NIPTMC & $4(16.66 \%)$ & $10(38.46 \%)$ & $9(25 \%)$ & $5(17.24 \%)$ & $9(17.64 \%)$ & $9(16.98 \%)$ & $6(18.75 \%)$ & $25(40.32 \%)$ & 27 (33.75\%) \\
\hline PTMC & $9(37.5 \%)$ & $13(50 \%)$ & $12(33.33 \%)$ & 9 (31.03\%) & $18(35.29 \%)$ & $16(30.18 \%)$ & $10(31.25 \%)$ & $31(50 \%)$ & $38(47.5 \%)$ \\
\hline All thyroid cancers & $24(100 \%)$ & $26(100 \%)$ & $36(100 \%)$ & $29(100 \%)$ & $51(100 \%)$ & 53 (100\%) & 32 (100\%) & 62 (100\%) & 80 (100\%) \\
\hline
\end{tabular}

IPTMC incidental papillary thyroid microcarcinoma, NIPTMC non-incidental papillary thyroid microcarcinoma, PTMC papillary thyroid microcarcinoma 


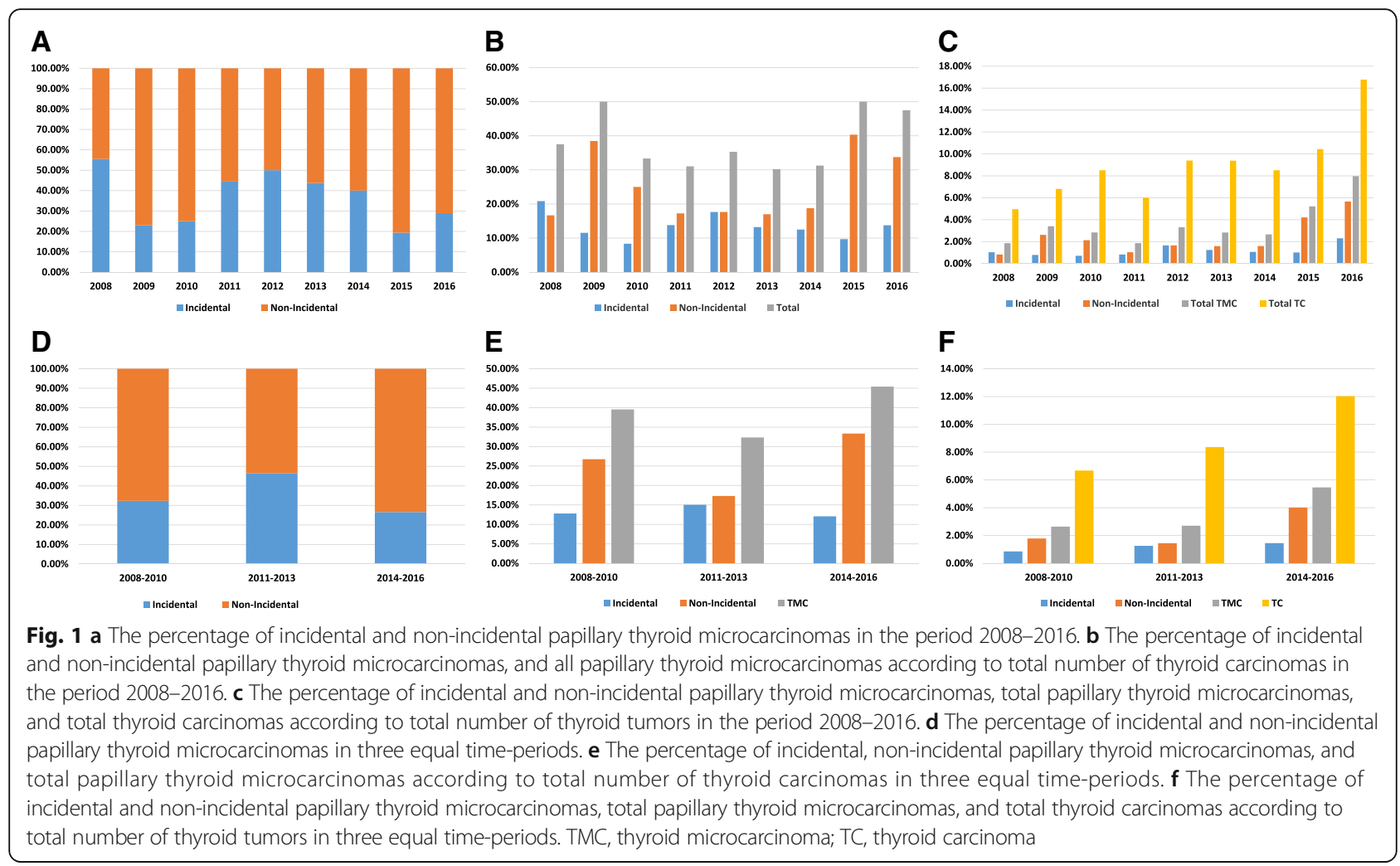

nodules [19]. Some authors have stated that the increasing rate of the prevalence of non-incidental thyroid microcarcinoma (NIPTMC) during the past few decades may have been due to the extensive development of high-frequency ultrasonography and the UG-FNAB technique [20]. These observations are in accordance with the results of our study. At the beginning of this trial, in 2008 , only $0.83 \%$ of all thyroid tumors were NIPTMC, whereas this figure was $5.66 \%$ in 2016.
The mortality rate of PTMC has remained unchanged over the last few decades, which additionally supports the hypothesis of increased NIPTMC diagnoses and treatment [21]. At present, even tumors with a 3-mm diameter can be detected by ultrasonography and subsequently qualify for UG-FNAB [15]. For this reason, a large number of very small malignant tumors are found, thus increasing the rate of NIPTMC. Chen et al. suggested that even nodules with a dimension of

Table 4 Patients with incidental and non-incidental papillary thyroid microcarcinoma, all thyroid cancers and all papillary thyroid microcarcinoma cases in three equal time-periods

\begin{tabular}{|c|c|c|c|}
\hline NIPTMC & $52(33.3 \%)$ & & \\
\hline \multirow[t]{2}{*}{ IPTMC } & $104(66.7 \%)$ & & \\
\hline & Period I (2008-2010) & Period II (2011-2013) & Period III (2014-2016) \\
\hline \multicolumn{4}{|c|}{ Number of cases in each period $[n]$} \\
\hline NIPTMC & 23 & 23 & 58 \\
\hline IPTMC & 11 & 20 & 21 \\
\hline TC & 86 & 133 & 174 \\
\hline \multicolumn{4}{|c|}{ Percentage of PTMC in all TC cases in each period [\%] } \\
\hline NIPTMC & $26.74 \%$ & $17.29 \%$ & $33.33 \%$ \\
\hline IPTMC & $12.79 \%$ & $15.04 \%$ & $12.07 \%$ \\
\hline PTMC & $39.53 \%$ & $32.33 \%$ & $45.40 \%$ \\
\hline$p$ & 0.0216 & 0.6176 & $<0.0001$ \\
\hline
\end{tabular}

Statistically significant differences are shown in italics

IPTMC incidental papillary thyroid microcarcinoma, NIPTMC non-incidental papillary thyroid microcarcinoma, PTMC papillary thyroid microcarcinoma, TC all thyroid cancers 
Table 5 Comparison of the three equal periods of time according to incidence of non-incidental and incidental papillary thyroid microcarcinoma

\begin{tabular}{llll}
\hline & $p$ & & \\
\cline { 2 - 4 } & Period I (2008-2010) & Period II (2011-2013) & Period III (2014-2016) \\
\hline Period I (2008-2010) & - & 0.0882 & 0.2804 \\
Period II (2011-2013) & 0.0882 & - & 0.0014 \\
Period III (2014-2016) & 0.2804 & 0.0014 & - \\
\hline
\end{tabular}

Statistically significant differences are shown in italics

2-3 $\mathrm{mm}$ can be detected with the use of a high-resolution transducer [19]. Comparing the rates of NIPTMC to those of IPTMC in the equally divided time-periods of our study, we noticed a statistically significant increase of NIPTMC rate in the last period. In those years, even very small tumors with suspicions of malignancy qualified for UG-FNAB, followed by early radical surgery [16]; consequently, the "wait and see" approach until the tumor had increased in size was rejected [22, 23]. We propose that this is the reason for the continuous and significant increase in NIPTMC diagnoses. All of the patients admitted to our clinic for thyroid tumors, who subsequently underwent surgical treatment, also underwent UG-FNAB. This diagnostic method was widely used to distinguish PTMC from benign tumors. Nevertheless, not every PTMC diagnosed postoperatively had been biopsied before surgical treatment; thus, not every "suspicious" tumor was selected for biopsy. This observation explains the fact that, even in period III (2014-2016), in which high-quality UG-FNAB was available, we still observed $12.07 \%$ of IPTMC. This phenomenon may also be explained by the observation of Brito et al. [21], who noticed that increasing rates of thyroid operations were coupled with more radical surgical treatment. We used a more radical treatment strategy, and it may justify the high incidence rate of IPTMC [21]. Castro et al. [24] concluded that the major reason for the increasing incidence of NIPTMC is the detection of subclinical, indolent tumors, thus, overdiagnosis. Regarding the indolent type of NIPTMC, it has been suggested to change the term "carcinoma" to "small papillary lesions." [24] This might be more suitable for patients in terms of the overdiagnosis and overtreatment phenomenon.

Our study has certain limitations. Firstly, this was a retrospective study and included patients who underwent UG-FNAB and thyroid surgery during a relatively not very long time. Secondly, we could not perform lymph nodes status analysis, because of retrospective study design. Patients with IPTMC did not received central lymph node dissection, because the cancer diagnosis was established postsurgery. And finally, the study included a relatively small number of patients. Unfortunately, surgery still not only represents a treatment option but also is a diagnostic tool in thyroid pathology.
Microscopic evaluation of the surgical specimen still represents the gold standard for the diagnosis of thyroid nodules. In regards to overdiagnosis and overtreatment, further studies are necessary to resolve these issues.

\section{Conclusions}

The proportion of IPTMC and NIPTMC of all thyroid tumors is relatively high and is increasing. However, in terms of total TCs, only the prevalence of NIPTMC, but not that of IPTMC, is increasing; this may be explained by better presurgical diagnostic processes.

\section{Abbreviations \\ IPTMC: Incidental papillary thyroid microcarcinoma; MNG: Multinodular goiter; NIPTMC: Non-incidental papillary thyroid microcarcinoma; PTC: Papillary thyroid cancer; PTMC: Papillary thyroid microcarcinoma; TC: Thyroid cancer; TT: Thyroid tumor; UG-FNAB: Ultrasound-guided fine-needle aspiration biopsy}

\section{Availability of data and materials \\ Not applicable.}

\section{Authors' contributions}

KK is responsible for the conceptualization, investigation, methodology, project administration, resources, writing the original draft, and reviewing and editing the manuscript. KK, PK, JM, AK, and OK obtained the data. KK and AZK did the formal analysis, supervision, and validation. All authors read and approved the final manuscript.

\section{Ethics approval and consent to participate}

All research was carried out in compliance with the Helsinki Declaration. This study was approved by the Ethics Committee of Wroclaw Medical University (number: KB-27/2018). The data were analyzed anonymously and retrospectively on the basis of medical records. The authors did not have access to any identifying patient information and did not have any direct access to the study participants.

\section{Consent for publication \\ Not applicable.}

\section{Competing interests}

The authors declare that they have no competing interest.

\section{Publisher's Note}

Springer Nature remains neutral with regard to jurisdictional claims in published maps and institutional affiliations.

\section{Author details}

${ }^{1}$ First Department and Clinic of General, Gastroenterological, and Endocrine Surgery, Wroclaw Medical University, 66 Maria Skłodowska-Curie Street, 50-369 Wrocaw, Poland. ${ }^{2}$ Department of Endocrinology and Diabetology for Children and Adolescents, Wroclaw Medical University, Wroclaw, Poland. 
Received: 31 August 2018 Accepted: 28 September 2018

Published online: 10 October 2018

\section{References}

1. Hu D, Zhou J, He W, et al. Risk factors of lateral lymph node metastasis in cN0 papillary thyroid carcinoma. World J Surg Oncol. 2018;16:30.

2. Are C, Shaha AR. Anaplastic thyroid carcinoma: biology, pathogenesis, prognostic factors, and treatment approaches. Ann Surg Oncol. 2006;13:453-64

3. Jemal A, Siegel R, Xu J, Ward E. Cancer statistics, 2010. CA Cancer J Clin. 2010;60:277-300

4. Magreni A, Bann DV, Schubart JR, Goldenberg D. The effects of race and ethnicity on thyroid cancer incidence. JAMA Otolaryngol Head Neck Surg. 2015;141:319-23.

5. Lu J, Hu S, Miccoli P, et al. Non-invasive diagnosis of papillary thyroid microcarcinoma: a NMR-based metabolomics approach. Oncotarget. 2016;7: 81768-77.

6. Hedinger C, Williams ED, Sobin LH. The WHO histological classification of thyroid tumors: a commentary on the second edition. Cancer. 1989; 63:908-11.

7. Zhao Q, Ming J, Liu C, et al. Multifocality and total tumor diameter predict central neck lymph node metastases in papillary thyroid microcarcinoma. Ann Surg Oncol. 2013;20:746-52.

8. Mehanna H, Al-Maqbili T, Carter B, et al. Differences in the recurrence and mortality outcomes rates of incidental and nonincidental papillary thyroid microcarcinoma: a systematic review and meta-analysis of 21329 personyears of follow-up. J Clin Endocrinol Metab. 2014:99:2834-43.

9. Solares CA, Penalonzo MA, Xu M, Orellana E. Occult papillary thyroid carcinoma in postmortem species: prevalence at autopsy. Am J Otolaryngol. 2005;26:87-90

10. Harach HR, Franssila KO, Wasenius VM. Occult papillary carcinoma of the thyroid. A "normal" finding in Finland. A systematic autopsy study. Cancer. 1985:56:531-8.

11. Chow SM, Law SC, Chan JK, Au SK, Yau S, Lau WH. Papillary microcarcinoma of the thyroid - prognostic significance of lymph node metastasis and multifocality. Cancer. 2003;98:31-40.

12. Gao X, Zhang X, Zhang Y, Hua W, Maimaiti Y, Gao Z. Is papillary thyroid microcarcinoma an indolent tumor?: a retrospective study on 280 cases treated with radioiodine. Medicine (Baltimore). 2016;95:e5067.

13. Nikiforov YE, Steward DL, Robinson-Smith TM, et al. Molecular testing for mutations in improving the fine-needle aspiration diagnosis of thyroid nodules. J Clin Endocrinol Metab. 2009;94:2092-8.

14. Haugen BR, Alexander EK, Bible KC, et al. 2015 American Thyroid Association Management Guidelines for adult patients with thyroid nodules and differentiated thyroid cancer: the American Thyroid Association Guidelines Task Force on Thyroid Nodules and Differentiated Thyroid Cancer. Thyroid. 2016;26:1-133.

15. Shi C, Guo Y, Lv Y, et al. Clinicopathological features and prognosis of papillary thyroid microcarcinoma for surgery and relationships with the BRAFV600E mutational status and expression of angiogenic factors. PLoS One. 2016;11:e0167414

16. Kaliszewski K, Wojtczak B, Strutyńska-Karpińska M, Łukieńczuk T, Forkasiewicz Z, Domosławski P. Incidental and non-incidental thyroid microcarcinoma. Oncol Lett. 2016:12:734-40.

17. Li B, Zhang Y, Yin P, Zhou J, Jiang T. Ultrasonic features of papillary thyroid microcarcinoma coexisting with a thyroid abnormality. Oncol Lett. 2016;12 2451-6.

18. Kaliszewski K, Zubkiewicz-Kucharska A, Wojtczak B, Strutyńska-Karpińska M, Zaleska-Dorobisz U, Leśków E. Ultrasound guided fine-needle aspiration biopsy of thyroid nodules: does radiologist assistance decrease the rate of unsatisfactory biopsies? Adv Clin Exp Med. 2016:25:93-100.

19. Chen HY, Liu WY, Zhu H, et al. Diagnostic value of contrast-enhanced ultrasound in papillary thyroid microcarcinoma. Exp Ther Med. 2016;11:1555-62.

20. Yoon JH, Lee HS, Kim EK, et al. Short-term follow-up US leads to higher false-positive results without detection of structural recurrences in PTMC. Medicine (Baltimore). 2016:9:e2435.

21. Brito JP, Davies $L$. Is there really an increased incidence of thyroid cancer? Curr Opin Endocrinol Diabetes Obes. 2014;21:405-8.

22. Ito $Y$, Miyauchi $A$, Inoue $H$, et al. An observational trial for papillary thyroid microcarcinoma in Japanese patients. World J Surg. 2010;34:28-35.
23. Kim HY, Park WY, Lee KE, et al. Comparative analysis of gene expression profiles of papillary thyroid microcarcinoma and papillary thyroid carcinoma. J Cancer Res Ther. 2010;6:452-7.

24. Castro MR, Morris JC, Ryder M, Brito JP, Hay ID. Most patients with a small papillary thyroid carcinoma enjoy an excellent prognosis and may be managed with minimally invasive therapy or active surveillance. Cancer 2015:121:3364-5.
Ready to submit your research? Choose BMC and benefit from:

- fast, convenient online submission

- thorough peer review by experienced researchers in your field

- rapid publication on acceptance

- support for research data, including large and complex data types

- gold Open Access which fosters wider collaboration and increased citations

- maximum visibility for your research: over $100 \mathrm{M}$ website views per year

At $\mathrm{BMC}$, research is always in progress.

Learn more biomedcentral.com/submissions 